\title{
Virtualization Software Applications
}

\author{
Meshal F. Aldhamen \\ Public Authority and Applied Education, High Institute of Telecommunication and Navigation, \\ Computer Department, Kuwait
}

\begin{abstract}
Virtualization can be seen as a significant part of a general example in big business IT that incorporates autonomic registering, a situation where in the IT condition will in all likelihood manage itself reliant on saw movement, and utility figuring, in which PC preparing force is viewed as an utility that customers can pay for similarly as required. The typical objective of virtualization is to unify regulatory missions while improving adaptability and undertaking. Different software has been installed in The Higher Institute of Telecommunications \& Navigation laps, some software is missing in some PC's, needs upgrade and reinstallation. This research will review one of Application virtualization arrangement that permits multiuser remote access to local programming virtualization server from any gadget, anyplace, whenever and utilizing different platforms.
\end{abstract}
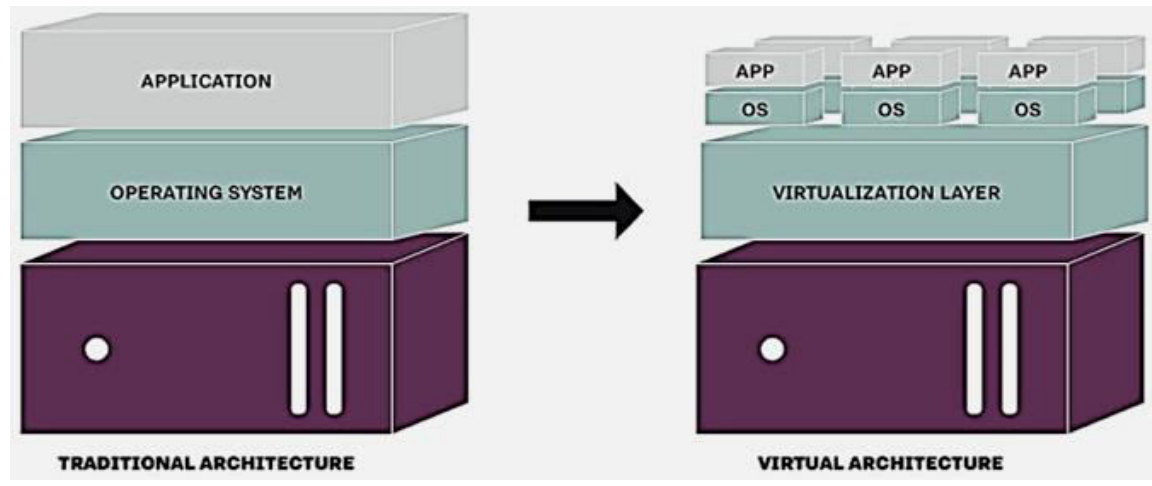

Figure 1:Before and after Virtualization

By creating this server, all instructors and students can use the updated software from the central server whether in LAPs Pc's or from instructor private PC's or gadgets. As a result, this virtualized server will solve software inaccessibility and defective issued in all LAPs PC's will likewise permit all organization segments to remotely associate with this server and utilize the software, will defiantly reduce the effort and cost to software installation and maintenance.

Keywords: Virtualization Software, Vmware, Hyper-V, Vsphere, Citrix Hypervisor

\section{INTRODUCTION}

Among the main business difficulties standing up to CIOs and IT, administrators today are savvy use of IT infrastructure; responsiveness in supporting new business activities; and adaptability in adjusting to authoritative changes. Driving an additional sense of urgency is the proceeded with the atmosphere of IT spending limitations and increasingly stringent administrative necessities. Virtualization is a fundamental technological innovation that enables skilled IT managers to convey imaginative answers for such business challenges. Virtualization programming portrays an innovation wherein an application, client working framework or information stockpiling is detached a long way from the veritable key equipment or programming.

A key use of virtualization innovation is server virtualization, which uses a product layer called a hypervisor to impersonate the concealed equipment. This often joins the CPU's memory, I/O and network traffic. The client working structure, consistently connecting with genuine equipment, is by and by doing all things considered with a product imitating of that equipment, and regularly the client working framework does not understand it's on virtualized equipment. While the display of this virtual structure isn't ascend to the introduction of the working system running on evident equipment, the idea of virtualization works considering the way that most client working systems and applications need not sit around idly with the full use of the fundamental equipment. This takes into account more prominent adaptability control and segregation by evacuating the reliance on a given hardware platform. While at first implied for server virtualization, the idea of virtualization has spread to applications, networks, data, and desktops. 
Is the most dominant answer for running Windows and Windows applications on Mac with fundamentally less disk, memory, and CPU usage. It empowers Mac clients to run Windows close by their local Operating System, for those moments when they need application that only works on that system. Parallels make Windows alarms show up in the Macintosh notification centre and work a brought together clipboard. Most Mac users consider Parallels an apparatus only for utilizing Windows, however it tends to be utilized to host a large scope of Linux, Chrome Operating System and other versions of Macintosh Operating System.

\section{ORACLE VM VIRTUAL BOX}

Is an amazing x86 and AMD64/Intel64 virtualization item for big business just as home use. Not exclusively is VirtualBox an amazingly highlight rich, elite item for big business clients, it is additionally the main expert arrangement that is unreservedly accessible as Open Source Software under the particulars of the GNU General Public License (GPL) rendition 2. By and by, VirtualBox keeps running on Windows, Linux, Macintosh, and Solaris has and underpins an enormous number of visitors working frameworks.

VirtualBox is as a rule effectively created with continuous discharges and has a regularly developing rundown of highlights, supported guest operating systems and platforms it runs on. VirtualBox is a network exertion sponsored by a devoted organization: everybody is urged to contribute while Oracle guarantees the item dependably meets proficient quality criteria.

Clients of VirtualBox can stack various visitor OSs under a solitary host working framework (have OS).

Every visitor can be begun, delayed and halted autonomously inside its own one of a kind virtual machine (VM). The customer can openly arrange each VM and run it under a decision of programming-based virtualization or equipment helped virtualization if the fundamental host equipment bolsters this. The host OS and visitor OSs and applications can talk with each other through different instruments including a run of the mill clipboard and a virtualized organize office. Visitor VMs can in like manner clearly talk with each other at whatever point arranged to do as such.

\section{VMWARE FUSION AND WORKSTATION}

VMware offers a far-reaching determination of virtualization products, with Fusion 10 for the Apple Mac and Workstation 14 for the PC. Despite the name difference, these two products offer adequately a similar arrangement, however custom fitted to each host OS. For the Mac that incorporates a slick 'Unity Mode' that empowers Mac OS to dispatch Windows applications from the Dock and have them seem like they're part of the host OS. Workstation, as the version numbering suggests, is a full-grown product and conveys a standout amongst the most advanced VM executions seen up until this point. Being one of only a handful couple of hosts that supports DirectX 10 and OpenGL 3.3, it permits CAD and other GPU accelerated applications to work under virtualization. VMware Workstation is a hosted hypervisor that runs on x64 versions of Windows and Linux working frameworks; it enables users to set up virtual machines on a single physical machine and use them all the while alongside the real machine. VMware Workstation Pro enables you to run various running frameworks without a moment of lost times on similar Operating Systems.

Create real Linux and Windows VMs and other desktops, server, and tablet environments, complete with configurable virtual networking, application testing, product demonstrations and that's just the beginning. Securely connect with vSphere, ESXi or other Workstation servers to dispatch, control and oversee both VMs and physical hosts. A typical VMware hypervisor maximizes productivity and empowers simple exchange of virtual machine to and from your local PC. Workstation Pro supports many working frameworks and run with cloud and holder technologies. Run a secure second desktop with various privacy settings, tools, and networking configurations, or utilize criminological tools to explore OS vulnerabilities. Workstation gives a standout amongst the most secure hypervisors in the business and conveys incredible highlights for IT security experts.

\section{VSPHERE}

Gives an astonishing, versatile, and secure foundation for business mastery that quickens your computerized change to half breed cloud and accomplishment in the advanced economy. With vSphere, you can bolster new outstanding burdens and use cases while keeping pace with the developing needs and multifaceted nature of your framework. vSphere is the centre of a protected SDDC, verifying applications, information, foundation, and access. Pushed security limits totally organized into the hypervisor and fuelled by AI, give better penetrability, confirmation and speedier response time for security rates. vSphere urges you to run, oversee, interface and secure your applications in an ordinary working condition over the half breed cloud. Streamline server farm tasks and the board at scale, increase business capability, and lessening CapEx and OpEx costs through virtualization. Connect on-premises conditions to 
vSphere-based open mists, for example, VMware Cloud on AWS, and value a streamlined half breed cloud understanding. Secure applications, framework, information, and access with far reaching, worked in security engineering and an operationally fundamental approach driven model. Secure the decency of your applications running on vSphere using AI and conduct assessment to screen against risks and mechanize responses. Give indications of progress penetrability and confirmation with a direct, versatile security game plan, with no authorities to manage and insignificant overhead. Improve the exactness and speed of acknowledgment, assessment, and time to respond to security perils. Exploit the most recent innovation advancements in equipment to turbocharge the presentation of existing applications. Bolster new outstanding tasks at hand and use cases, for example, AI, man-made brainpower, huge information, and elite registering. Balance workloads and organize asset allotment to guarantee top execution for your basic applications. Arrangement and deploy workloads consistently over the hybrid cloud environment. Cross over any barrier among IT and developers with vSphere Integrated Containers.

Utilizing the centre capacities of vSphere, your IT groups can run both customary and containerized applications one next to the other on a similar foundation, without the requirement for extra tooling or preparing. Streamline your enormous data infrastructure management while making it more cost practical. VMware is the best platform for huge information, similarly all things considered for customary applications. Manage your remote offices and branch offices with practically or no local IT staff. Empower fast provisioning of servers through virtualization, minimization of host arrangement float, and improved permeability into administrative consistency, over different sites. Migrate IT inheritance Unix infrastructure to virtualized Linux. Get elite, improved accessibility, bigger VM capacity, and debacle recuperation abilities.

\section{QEMU}

Is a conventional and open source machine emulator and virtualizes. At the point when utilized as a machine emulator, QEMU can run OSes and programs made for one machine (e.g. an ARM board) on an alternate machine (e.g. your own PC). By utilizing dynamic interpretation, it accomplishes generally excellent execution. When utilized as a virtualize, QEMU accomplishes close execution by running the client code legitimately on the host Central Processor Unit. QEMU bolsters virtualization when running under the Xen hypervisor or utilizing the unit piece module in Linux. When utilizing KVM, QEMU can virtualize x86, server and installed PowerPC. It figures out how to do this without administrator privileges, and the performance of VMs running on it is near that of native establishments. What QEMU needs is any refined interface apparatuses, rather depending on CLI contributions to introduce and design VM customers. As of now, it is additionally just ready to host on Linux, regardless of whether it can run a wide scope of working frameworks under that.

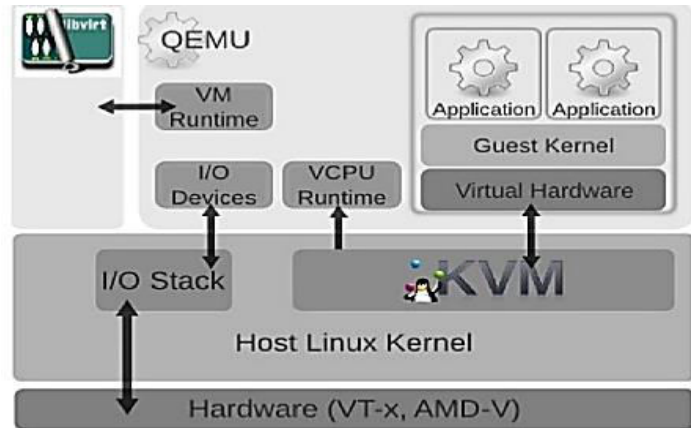

Figure 2: Virtualization Architecture

VII.

RED HAT VIRTUALIZATION

Kernel-based Virtual Machine is an open source virtualization technology incorporated with Linux®. In particular, Kernel-based Virtual Machine gives you a chance to convert Linux into a hypervisor that allowed a host machine to work, isolated virtual environments called client or VMs.

Kernel-based Virtual Machine is part of Linux. In the event that you have more current Linux, you have Kernel-based Virtual Machine. KVM was 1st declared in 2006 and converged into the primary Linux versions after one year. Since Kernel-based Virtual Machine is a piece of current Linux code, it promptly profits by each new Linux highlight, fixes, and headway without extra designing. KVM changes over Linux into a kind one (uncovered metal) hypervisor.

All hypervisors need some working system level portions, for instance, a memory boss, process programmed, input/yield (I/O) stack, device drivers, security chairman, a system stack, and anything is possible from that point- to work Virtual Machines. Kernel-based Virtual Machine has all of these portions since it's a piece of the Linux part.

Each Virtual Machine is realized as a customary Linux procedure, booked by the default Linux scheduler, with committed virtual equipment like a system card, designs connector, CPU(s), memory, and circles. Long story short, you need to run an adaptation of Linux that was discharged after 2007 and it should be installed on X86 hardware that 
supports virtualization abilities. In the event that both of those containers are checked, at that point you should simply stack 2 existing modules (a host kernel module and a processor- explicit module), an emulator, and any drivers that will enable you to run extra frameworks. Building a virtual framework on a stage you're legally attached as far as possible your entrance to the source code. That implies your IT developments are most likely going to be more workarounds advancements, and the following contract could shield you from putting resources in clouds, containers, and automation. Relocating to a KVM-based virtualization stage means having the option to investigate, alter and improve the source code behind your hypervisor. What's more, there's no enterprise-license understanding on the grounds that there's no source code to ensure. It's yours. It's conceivable to physically deal with a bunch of VM started up on a solitary workstation without an administration tool. Huge companies use virtualization the board programming that interfaces with virtual situations and the principal physical equipment to adjust asset organization improve data assessments and streamline exercises. Red Hat made Red Hat Virtualization for exactly this explanation.

\section{MICROSOFT HYPER-V}

Despite whether you are a programmer, an IT proficient, or a technology enthusiast, a noteworthy number of you must run various working frameworks. Hyper-V gives you a chance to run various working frameworks as VM on Windows. Hyper-V explicitly gives hardware virtualization. That implies each virtual machine keeps running on virtual hardware. Hyper- $\mathrm{V}$ gives you a chance to make virtual hard drives, virtual switches, and various other virtual gadgets which can all be added to virtual machines. Hyper- $\mathrm{V}$ is accessible on 64-bit renditions of Windows 10 Pro, Enterprise, and Education. It isn't accessible on the Home edition. Most computers will run Hyper-V anyway each virtual machine is a totally independent working framework. You can, for the most part, run at least one virtual machine on a PC with 4GB of RAM, however, you'll require more assets for extra virtual machines or to install and run resource extreme software like games, video editing, or engineering design software.

Hyper-V on Windows supports a wide range of working frameworks in a virtual machine including different arrivals of Linux, FreeBSD, and Windows. There are a few highlights that work diversely in Hyper-V on Windows than they do in Hyper-V running on Windows Server. Hyper-V features only available on Windows 10 and Server. The memory management model is distinctive for Hyper-V on Windows. On a server, Hyper-V memory is dealt with the supposition that lone the VM are working on the server. In Hyper-V on Windows, memory is made to do with the desire that most customer workstations are working applications on host notwithstanding running VM. Projects that rely upon explicit equipment won't function admirably in a VM. For instance, amusements or software that require handling with GPUs probably won't function admirably. Likewise, applications depending on sub-10ms clocks, for example, unrecorded music blending software or high exactness times could have issues running in a VM. What's more, on the off chance that you have Hyper-V empowered, that dormancy touchy, high-exactness applications may likewise have issues running in the host. This is claiming with virtualization empowered, the host OS additionally keeps running over the Hyper- $\mathrm{V}$ virtualization layer, similarly, as guest working frameworks do. In any case, in contrast to guests, the host OS is extraordinary in that it has direct access to all the hardware, which implies that applications with uncommon hardware prerequisites can even now keep running without issues in the host OS.

\section{CITRIX HYPERVISOR}

Citrix Hypervisor is the main virtualization the executives stage enhanced for application, desktop and server virtualization foundations. Solidification and control of workloads on Citrix Hypervisor empower associations of any vertical or size to change their business IT process foundations. Citrix Hypervisor is a complete server virtualization stage with enterprise-class highlights worked in to effectively deal with various remaining burden types, blended working frameworks, and capacity or networking configurations. For the most requesting application, and desktop virtualization use cases, its industry-driving versatility, and execution under the burden can take into account the biggest Citrix Virtual Apps and Desktops arrangements. IT gets the advantage of highlights one of a kind to Citrix Hypervisor, for example, upgraded virtualized graphics with NVIDIA and Intel, and improved workload security with Direct Inspect APIs, all of which diminish virtual foundation expenses and unpredictability.

Citrix Virtual Apps and Desktops license holders can apply for those item licenses legitimately to Citrix Hypervisor foundation by means of the license server; no different or explicit Citrix Hypervisor license is required. Citrix Hypervisor empowers IT to merge and contain a critical number of data centre servers. This builds generally speaking use of existing hardware assets while bringing down foundation absolute expense of ownership. Administrators can expand efficiencies inside their framework, dispensing with arranged blackouts and limiting impromptu ones. Extensive server union and control with Citrix Hypervisor likewise take into consideration critical execution and limit gains in CPU cores, host RAM, VM RAM and virtual disks per VM, as well as joining with Microsoft Windows Update Services for automatic Windows VM driver updates. Citrix Hypervisor is the leader server virtualization platform offering help for elite improved 3D graphics, with the most stretched out assortment of GPU pass-through and virtualized GPU vendor options. Just Citrix Hypervisor incorporates support for Intel's Virtual Graphics Technology 
(GVT-g), a CPU installed GPU with no additional hardware required to encourage upgraded graphics workloads. Citrix Hypervisor backing of NVIDIA GRID vGPU has been stretched out for Linux graphical applications and work areas. With the most recent NVIDIA GRID Maxwell-gen hardware reconciliation, vGPU sessions per host have now expanded to 128 .

\section{CONCLUSION}

The benefits of changing to a virtual environment are copious, setting aside you cash and time while giving a lot of more prominent business coherence and capacity to recover from the debacle.

Decreased spending. Virtualizing requires fewer servers and expands the life expectancy of existing hardware. This additionally means decreased energy costs.

Simpler backup and catastrophe recovery. Virtualization makes recovery a lot swifter and precise, with less labour and a small amount of the equipment.

Better business progression. Virtualization gives representatives access to software, documents and interchanges anyplace they are and can empower various individuals to get to similar data for greater progression.

Progressively proficient IT tasks. Virtualization gives a simpler course to specialists to introduce and look after software, distribute updates and keep up a progressively secure network. They can do this with less personal time, fewer blackouts, faster recuperation, and moment reinforcement when contrasted with a non-virtual environment.

The disadvantages of virtualization are generally those that would accompany any technology transition. With cautious arranging and master usage, these disadvantages can be survived.

Upfront Expenses. The interest in the virtualization software and potentially extra hardware may be required to make the virtualization conceivable. This relies upon your current network. Numerous organizations have adequate ability to oblige the virtualization without requiring a ton of money. This obstruction can likewise be all the more promptly explored by working with a Managed IT Services supplier, who can counterbalance this expense with month to month renting or buy plans.

Software licensing contemplations. This is winding up to a lesser degree an issue as more software merchants adjust to the expanded reception of virtualization, yet it is essential to check with your sellers to obviously see how they see software use in a virtualized environment.

Possible learning curve. Actualizing and dealing with a virtualized environment will require IT staff with ability in virtualization. On the client side, a run of the mill virtual condition will work comparable to the non-virtual condition. There are a few applications that don't adjust well to the virtualized condition - this is something that your IT staff should know about and address before changing over.

For many businesses contrasting the focal points with the disservices, moving to a virtual environment is commonly the unmistakable champ. Regardless of whether the disadvantages present a few difficulties, these can be immediately explored with a specialist IT group or by re-appropriating the virtualization procedure to a Managed IT Services supplier. The appearing drawbacks are bound to be basic difficulties that can be explored and defeat effectively.

\section{REFERENCES}

[1]. (2016). What is virtualization? - Definition from WhatIs.com. Retrieved August 3, 2019, from https://searchservervirtualization.techtarget.com/definition/virtualization

[2]. J, C. G. J. (2017). Op het eerste gezicht Facsimile ISBN [Epub]. Lulu.com. Retrieved from https://www.vmware.com/pdf/virtualization.pdf

[3]. (2019, August 15). What's New in the Current Version?: Parallels Desktop 15 for Mac. Retrieved from https://www.parallels.com/products/desktop/whats-new/

[4]. What is VirtualBox?. (2019). Retrieved 2 September 2019,from https://www.linkedin.com/learning/learning-virtualbox/what-is-virtualbox

[5]. VMware. (2019, August 14). Server Virtualization Software |vSphere | VMware. Retrieved August 25, 2019, from https://www.vmware.com/mena/products/vsphere.html

[6]. QEMU version 4.1.0 User Documentation. (2019). Retrieved 4 July 2019, from https://qemu.weilnetz.de/doc/qemu-doc.html

[7]. Microsoft.(2018, June 25). Introduction to Hyper-V on Windows 10. Retrieved August 29, 2019, from https://docs.microsoft.com/enus/virtualization/hyper-v-on-windows/about/?ranMID $=24542$

[8]. citrix. (2019, May 16). Citrix Hypervisor powers a complete virtualization solution | Citrix Blogs. Retrieved August 25, 2019, from https:/www.citrix.com/blogs/2019/05/15/citrix-hypervisor-powers-a-complete-virtualization-solution/

\section{BIOGRAPHY}

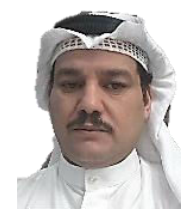

Professor Meshal Aldhamen Work for the higher Institute of Telecommunication \& Navigation. Department of Computer Technology, Kuwait.

Citrix Certified Administrator (CCA), Citrix Certified Instructor (CCI), A+ Certification, Empire WHO'S WHO Award. 